\title{
Concept for Robot-Based Cable Assembly Regarding Industrial Production
}

\author{
Daniel Gebauer, Jonas Dirr and Gunther Reinhart
}

\begin{abstract}
The assembly of cables in industrial production is still a largely manually performed task. Therefore, automatic cable assembly offers much potential in terms of efficiency. The major challenge of automating this task lies in the formlessness of the cables, which entails unknown and inconstant states of the assembly objects. In this paper, a process chain and a concept are presented for the automated cable assembly in an industrial context. The process chain consists of five process steps, which are used to structure existing approaches and system configurations for automated cable assembly from a production technology perspective. The emphasis is on the coverage of the process steps and the system technology. The presented concept represents an approach for robotic cable assembly focusing on the flexibility to process multiple product variants. Basis for the ability to handle a variety of variants is the avoidance of a forced shape on the cables. For this approach, system technology as well as challenges and possible solutions are presented.
\end{abstract}

\section{Keywords}

Assembly $\cdot$ Cable $\cdot$ Deformable objects $\cdot$ Gripper $\cdot$ Machine vision

D. Gebauer $(\bowtie) \cdot$ J. Dirr $(\bowtie) \cdot$ G. Reinhart Institute for Machine Tools and Industrial Management, Technical University of Munich, Boltzmannstr. 15, 85748 Garching, Germany e-mail: Daniel.Gebauer@iwb.tum.de

J. Dirr

e-mail: Jonas.Dirr@iwb.tum.de 


\section{Introduction}

The productivity and competitiveness of companies can be increased by automation and the use of industrial robots [1]. However, there are production processes that are difficult to automate due to technical reasons. One example is the production of control cabinets. The particular challenge is caused by the formlessness of the cables, which entails complex material behavior. In the production of control cabinets, wiring takes by far the most time [2]. Thus, automation of this process step offers great potential for increasing efficiency. Although a few robot-based approaches for cable assembly exist, there is currently no approach that takes into account all process steps ranging from the supply of cables to quality inspection. Therefore, we examine the robot-based cable assembly as a process chain from the point of view of production technology. Following this introduction, in Sect. 2 we present existing approaches in detail and discuss their coverage of the individual steps of the introduced process chain. Subsequently, we introduce our holistic concept for robot-based cable assembly in Sect. 3. Challenges and possible solutions are discussed, structured by the process steps. Section 4 summarizes this work and gives an outlook on further investigations.

\section{State of the Art}

This section aims to present the state of the art in the automated cable assembly using robot systems. First, commercial solutions for industrial applications are presented. Second, an existing structure for assembly functions is adapted to the robot-based cable assembly resulting in a five-step process chain. Present research approaches are described and classified concerning the process steps. Lastly, the state of the art is reflected critically and evaluated.

\subsection{Industrial Solutions}

So far, few commercial solutions exist for the automated assembly of cables. The wiring center Averex from Rittal GmbH \& Co. KG is a portal machine, which covers the control cabinet assembly from the preprocessing of the cables to the mounting in the cabinet terminal and the routing in the cable duct [3]. The cables are supplied within tubes to the end effector. Therefore, the system can only handle a limited range of cable diameters. Despite its comprehensive coverage of the cable assembly process, the system is limited to few specific use cases.

System Robot Automazione Srl offers the Universal Robotized Wiring System SYNDY [4]. It is an assembly system for ceiling lights based on a six-axis industrial robot and an end effector specifically designed for the following use case. The robotic system feeds the cables in the end effector to the gripper, where it can mount cables in two different types of terminals from both vertical and horizontal directions. The system seems 
to be limited due to its narrow use case in the lighting sector and the restriction to specific cable diameters.

Besides the commercial solutions mentioned above, cooperations of academia, research institutes, and industry exist. The Chair of Production Systems at the Ruhr University Bochum collaborates in the research project, RoboSchalt with SCHUBS $\mathrm{GmbH}$ [5]. In another project, Fraunhofer IPA and Rittal GmbH \& Co. KG have implemented the Automatic control cabinet cabling [6]. Both concepts address the automated cable assembly in control cabinets with two industrial robots, but only provide little information on details.

\subsection{Research Approaches}

In Lotter and Wiendahl [7], four assembly functions are presented: supply, handling, joining, and inspection. Due to the uncertain deformation of cables during handling, we propose to subdivide this step into grasping and manipulation, as cables are particularly complex here. Based on Lotter and Wiendahl [7], we propose a five-step process chain for the robot-based cable assembly as shown in Fig. 1.

The supply step represents the deployment at the very beginning of the assembly process. For an undefined supply pose, the initial object recognition and classification is part of the supply step. During grasping, manipulation, and joining, the cable is subjected to various external forces, e.g. applied by the gripper. In addition to gravity, these forces can influence the shape of a cable and thus make it more difficult to carry out the assembly. The inspection can be performed as a downstream process step or simultaneously to joining. In the following, the existing approaches are described in general terms and evaluated on which steps of the process chain the research is focused.

Table 1 summarizes this classification and shows the system technology that is used. The system technology is listed by three further subcategories: sensors, modeling, and gripping technology.

In Shirakawa et al. [8], an enhanced method for string shape recognition from 3D point clouds is presented. The method ranges from data acquisition and string shape recognition to the derivation of a point chain model. A detailed description of the procedure for the string shape recognition is provided, which demonstrates the extraction of a centerline point cloud from the 3D raw data. Subsequently, the point chain model is reconstructed from the point cloud. The described method is independent of the system technology, but

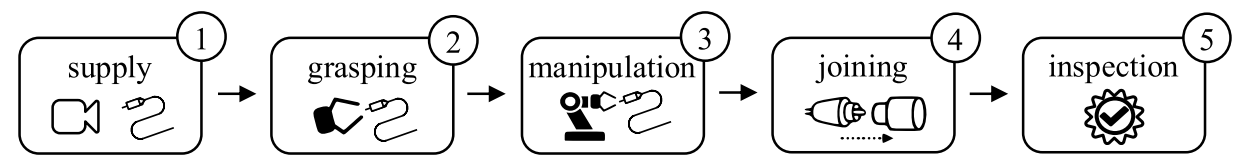

Fig. 1 Five-step process chain for robot-based cable assembly 


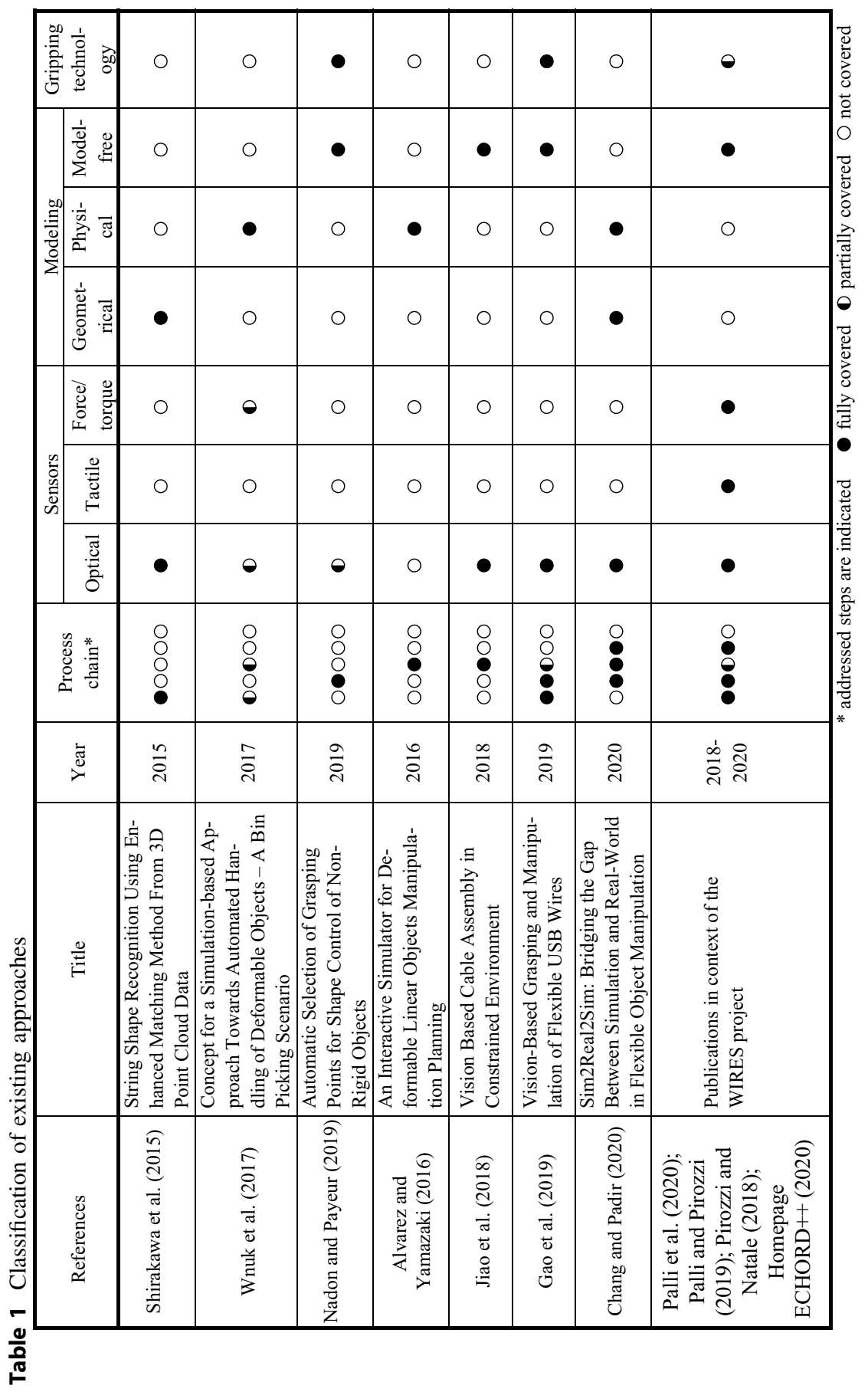


only requires the point cloud data of the string from multiple viewpoints. The paper focuses on the shape recognition of unknown deformable linear objects (DLOs) in uncertain conditions.

In Wnuk et al. [9], a conceptual design for an experimental setup for bin picking of deformable objects (DOs) is presented. First, the possibilities of modeling and simulating DOs are presented. The depiction of large deformations with simultaneous reduction of the calculation time was identified as the main requirement for the models. Finite elements methods and mass-spring systems do not match these requirements, whereas multi-body systems do so and therefore are considered the most appropriate. Secondly, a draft conceptual overview for localization, gripping, and manipulation of DOs is presented. Linking existing approaches from the research field of bin-picking with the above modeling approaches are currently being investigated.

In Nadon and Payeur [10], a strategy to control the shape of a DO within a gripper is presented. A depth-sensing camera is positioned almost vertically above a three-finger robot hand which is opened upwards. The user can initially specify the desired contour of the object. Thus, the model-free algorithm automatically generates grasping points and deforms the object into the desired shape.

In Alvarez and Yamazaki [11], an approach is presented that automatically parameterizes physical characteristics of DLOs through visual analysis in order to provide a simulation for manipulation planning. The method takes a set of pictures of a cable before and after a specific manipulation as input. From the pictures of both states, virtual representations are created based on a mass-spring system. The physical behavior of the DLOs is characterized by four synthetic parameters, which values are randomly assigned to each virtual representation of the DLO before the manipulation. Following, a specific manipulation is applied to the parameterized simulation. Based on the geometric distance between the resulting DLO of the manipulation task and the virtual representation of the second state, a difference score is computed. After several iterations with newly randomized parameters, the system selects the parameters with the best fitting score for application in manipulation planning.

In Jiao et al. [12], a model-free method for optically controlled cable routing in structured environments is presented. A cable is routed around pre-known obstacles to achieve a defined shape. Therefore, one cable end is gripped by the robot, while the other cable end is already joined. An industrial collaborative robot with a depth-sensing camera and a two-finger-gripper is used. The cable deformation is detected by markers attached to the cable.

In Gao et al. [13], the soldering of USB wires is described. Visual feedback is applied for the cable identification and sorting of the four cores of a USB cable. Based on the color, the cores are identified and the grasping points are computed, to move them in pre-defined grooves for soldering. In case a wire abandons the groove due to its elasto-plastic behavior, a compensation algorithm is triggered to re-grasp and re-place the wire in the grove. 
In Chang and Padir [14], a plug-in task of a household plug is addressed. The problem is approached using a 6 DOF assistive robot arm, two RGB-D cameras, and April tags. A new three-step procedure called Simulation-to-Real-to-Simulation is presented in order to address the gap between simulation and the real world. Firstly, a rough simulation model is created for the plug-in task. Secondly, the performance of the simulation is compared to the behavior in the real world. Lastly, based on the difference between both, the simulation is updated.

In Palli et al. [15], Palli and Pirozzi [16], Pirozzi and Natale [17], a holistic approach and prototype for automated assembly of cables in control cabinets are presented. For this purpose, an industrial robot with a custom end-effector, with multiple integrated sensors, was developed. The system utilizes a tactile sensor between the gripper jaws, force-torque sensing, and multiple optical sensors. 2D-cameras are used to detect and classify cables during the supply phase and terminals in the control cabinet. The tactile and optical sensor both estimate the pose of the cable tip, while the gripper manipulates it, to apply a pose correction for the joining. Besides, the system has a screwing unit and two linear actuators to screw down cables in the terminals. This work, as well as the prototype, has been developed in the context of the WIRES project [18] of the University of Bologna until the end of 2019. This project shows the largest coverage of the process chain among the compared approaches. The steps supply, grasping, and joining are fully addressed. Manipulation is considered with a reduced level of detail because mainly short cable segments are manipulated. An outlook on the inspection is given without going into further detail. The EU project REMODEL [19] takes up the topic and is going to continue it until 2023.

\subsection{Evaluation of the State of the Art}

Two main conclusions can be drawn from the state of the art. Firstly, there is currently no research approach that fully covers the five-step process chain. Moreover, half of the approaches presented address only one process step without aiming at production technology. Secondly, most of the industrial solutions apply a predicament on the cables, which means that the shape of the cable is restricted by the system technology. For instance, a predicament can be created through form closure, e.g. by feeding cables in tubes, or force closure, e.g. by multiple gripping points while the object is kept permanently under tension. Applying a predicament restricts unintentional movements of the cable during assembly. Nevertheless, cable feeding in tubes limits the system in terms of flexibility of variants and customized production. Also, multi-robot-systems with several gripping points stand out due to their high system complexity and effort for system tuning and therefore offer less flexibility, e.g. regarding supply. 


\section{Concept}

To address the limitations identified in 2.3, we propose an approach for robot-based cable assembly that covers the entire process chain and does not apply a predicament in order to increase the flexibility by extending the range of possible cable diameters and connector types. Therefore, a stereo vision sensor and a specifically designed end-effector will complement a $6 \mathrm{DOF}$ industrial robot. The main goal is to increase the product range, in the form of different cable diameters and connectors, of a robot-based cable assembly system in all five steps of the process chain and to reduce system complexity concurrently. This results in a system setup, which aims to be easily adaptable to multiple cable variants, diameters, connectors, and joining technologies. In the upcoming sections, we present our concept with respect to the five-step process chain. Thereby we will discuss the benefits of the proposed system as well as arising challenges and multiple solutions to address such.

\subsection{Supply}

The way goods are provided for assembly processes has to be adapted to the capabilities and degree of automation of the overall system. Especially semi-automated systems often require assistance, e.g. by a defined provision of the assembly products [15-17]. Compared to existing systems, the proposed approach uses only one grasping point. Therefore, the supply can be designed more flexibly, such that the cable can be provided in different ways, e.g. in an undefined shape on a planar surface, hanging, or in a fixture. The flexibilization of the supply offers two opportunities. On the one hand, the effort for the supply can be reduced by adjusting it to upstream process steps. On the other hand, a decoupling of upstream process steps such as pre-assembly is possible.

A flexible provision through an unknown location and form poses additional challenges to the system technology. When using optical sensor technology, challenges such as occlusion need to be considered, especially for cables with a knot-like structure and overlapping cable course. The occlusion can hide characteristic features of a cable such that pose estimation, object recognition, and gripper accessibility are complicated. The usage of 3D vision technology, as applied in Jiao et al. [12], allows a determination of the cable pose in space. Data acquisition from multiple points of view can inhibit occlusion with additional effort [8]. Optical markers and tools such as April tags can support object identification, but generate extra effort [14]. CAD-matching can be applied for pose measurement using the non-shape-labile components of a cable, such as connectors. 


\subsection{Grasping}

Following the initial recognition in the supply, the downstream process grasping can be addressed. While two-finger parallel grippers are used in many approaches (e.g. 4, 6, 13, $15,16,17,20)$, to the authors' knowledge no commercially available gripper exists, which is aimed at robot-based cable assembly. Furthermore, the design process of gripper systems for robot-based cable assembly has received little consideration in the literature so far. Thus, the following section describes the functionalities a gripper system must offer with regard to the further process steps.

Firstly, a gripper system must be able to grasp and later join a wide variety of cables and connectors. Secondly, the gripping pose depends on the shape of the cable or the type of connector as well as the type of joining connection in the downstream joining process. Unforeseeable changes in shape, which can occur e.g. during the gripping process or the manipulation, require once again the detection of the cable end or connector in order to enable a pose correction for the end-effector for the joining. So far, this requirement has mainly been addressed by the WIRES project [18] in which tactile and optical sensor technology is applied. Tactile sensor technology is integrated into the gripper jaws. Thus, the cable shape within the gripper jaws can be detected, and the protruding cable end or connector end can be extrapolated. As an alternative, 2D image data can be used to measure the shape of the protruding cable end or connector end. However, a stationary mounting of the vision sensor limits the flexibility or even the usability of the vision sensor for further functions such as the detection of cables and connectors during supply [15-17]. In order to be able to control the existing diversity of variants with a single gripper system, it is to be investigated how the insights gained in the WIRES project [18] for single core wires can be transferred to connectors and used for a comprehensive solution.

\subsection{Manipulation}

After the cable has been successfully grasped, it has to be manipulated into the joining pose. If the cable is not kept under permanent tension by multiple grippers like, e.g., in [6], but just by one gripper, there is a risk that the freely moving cable end will swing up and collide. Collisions can occur with the robot system, periphery elements, or the cable itself. In our approach, we aim to compensate for additional actuators, such as a second gripper, by using simulations and optical sensor technology.

Therefore, one suitable option is simulation-based offline trajectory planning in advance of the manipulation. Physical models are commonly used to describe the dynamical behavior of deformable objects, as e.g. in Wnuk et al. [9], Alvarez and Yamazaki [11], Arriola-Rios and Wyatt [21], Boonvisut and Cavuşoğlu [22]. Some of these approaches can be applied for robot-based cable assembly. The main disadvantage of this, however, is that model parameters of cables must be provided. 
Another possibility is to track the deformable object, e.g. with an optical sensor, as in Gao et al. [13], Matsuno et al. [23], or Leizea et al. [24]. This information could then be used to adjust the robot trajectory during the manipulation. This approach makes the determination of material parameters superfluous but imposes additional requirements on the system technology. On the one hand, there is high data processing effort and the need for real-time capability. On the other hand, it has to be ensured that the sensor has a clear view of the cable and that the permissible distance and angle between the sensor and the cable is maintained.

Therefore, another possibility is to automatically identify the material parameters of a cable in a first step by manipulating it along a specific trajectory and tracking its behavior with an optical sensor. In a second step, material parameters of the cable can be extracted from this data and used in a third step for offline path planning.

\subsection{Joining}

After the cable has been manipulated into the correct joining pose collision-freely, the cable end or connector has to be joined to the product. The goal of the proposed approach is to be able to assemble as many different cable variants as possible with one gripper system, as far as the joining processes are similar. An example of this is the joining of a single wire with an ultrasonically compressed strand into a spring-cage terminal as well as the joining of a coaxial plug into a corresponding socket using the same gripper system. Thus, a major challenge in joining is the filigree character. As the pose accuracy of commercially available, conventional industrial robots is in the range of several millimeters [25], they may be too imprecise for joining specific cable variants. In addition, the cable shape, at least the cable end or the connector end, respectively, has to be determined precisely before joining. Integrating tactile sensor technology directly into the gripper jaws or applying optical sensor technology to measure the protruding cable end [15-17] or connector end, as described in grasping, seem to be the most promising approaches. However, it has to be considered that optical sensory can compensate the pose inaccuracy of the robot, whereas this is not possible for tactile sensory. Therefore, the exclusive use of the latter may still be too imprecise for some specific cable variants.

\subsection{Inspection}

Although joining is the final process step that directly contributes to value creation, quality inspection is very important for automating cable assembly to check and record production quality. Manual joining can be prone to errors since many electronic products, such as control cabinets, have a highly complex structure. In practice, employees execute the quality control during the assembly process by pulling each cable to testing if the cable is sufficiently fixed. In scientific papers, both completeness control and quality inspection 
have been treated very little for automatic cable assembly. To automate this step, two main possibilities are seen. Firstly, 2D or 3D camera technology can be used for a visual completeness check for electronic products. Secondly, an automated quality control of the cable connection during the joining process is feasible. Similar to the quality control carried out by employees, a combination of optical data and force data can be used to assess the correctness of the joining process automatically.

\section{Conclusion and Outline}

In this paper, a holistic five-step process chain for robot-based cable assembly in industrial production was presented. Based on this process chain, existing approaches for automated cable assembly were examined and for the first time analyzed from a production technology perspective. Furthermore, a concept for robot-based cable assembly was presented, which increases the degree of assembly flexibility by waiving the predicament of the cable during assembly. The focus of the concept was on challenges and possible solutions regarding the five steps. Our future work will aim to implement the possible solutions identified in the concept.

Acknowledgements The research leading to this publication has received funding from the Bavarian Ministry of Economic Affairs, Regional Development, and Energy (StMWi), as part of the project "RoMaFo-Roboterassistenzsystem und maschinelles Sehen zur Montage von formlabilen Bauteilen bei kundenindividuellen Produkten" (DKI0109/01).

\section{References}

1. Alvarez, N., Yamazaki, K.: An interactive simulator for deformable linear objects manipulation planning. In: IEEE International Conference on Simulation, Modeling, and Programming for Autonomous Robots (2016)

2. Arriola-Rios, V.E., Wyatt, J.L.: A multimodal model of object deformation under robotic pushing. IEEE Trans. Cogn. Dev. Syst. (2017)

3. Boonvisut, P., Cavușoğlu, M.C.: Estimation of soft tissue mechanical parameters from robotic manipulation data. IEEE/ASME Trans. Mechatron. (2013)

4. Chang, P., Padır, T.: Sim2Real2Sim: Bridging the Gap Between Simulation and Real-World in Flexible Object Manipulation (2020)

5. Cordis Homepage: Robotic Technologies for the Manipulation of Complex DeformablE Linear objects. https://cordis.europa.eu/project/id/870133/de. Accessed 6 Sept 2020

6. Fraunhofer IPA Homepage. https://www.pitasc.fraunhofer.de/en/application.html. Accessed 6 Sept 2020

7. Gao, Y., Chen, Z., Liu, Y.-H.: Vision-based grasping and manipulation of flexible USB wires. In: Proceeding of the IEEE International Conference on Robotics and Biomimetics (2019)

8. Homepage ECHORD++: WIRES-Wiring Robotic System for Switchgears. https://echord.eu/ wires.html. Accessed 6 Sept 2020

9. IFR Statistical Department: World Robotics 2018 (2018) 
10. Jiao, C., Jiang, X., Li, X., Liu, Y.: Vision based cable assembly in constrained environment. In: IEEE International Conference on Robotics and Biomimetics (2018)

11. LSP RUB Homepage. https://www.lps.rub.de/forschung/projekte/roboschalt. Accessed 6 Sept 2020

12. Leizea, I., Mendizabal, A., Alvarez, H., Aguinaga, I., Borro, D., Sanchez, E.: Real-time visual tracking of deformable objects in robot-Aasisted surgery. IEEE Comput. Graph. Appl. (2017)

13. Lotter, B., Wiendahl, H.-P.: Montage in der Industriellen Produktion. Springer-Verlag, Berlin (2012)

14. Matsuno, T., Tamaki, D., Arai, F., Fukada, T.: Manipulation of deformable linear objects using knot invariants to classify the object condition based on image sensor information. IEEE/ASME Trans. Mechatron. (2006)

15. Nadon, F., Payeur, P.: Automatic selection of grasping points for shape control of non-rigid objects. In: IEEE International Symposium on Robotic and Sensors Environments (2019)

16. Palli, G., Pirozzi, S., Indovini, M., Gregorio, D. de, Zanella, R., Melchiorri, C.: Automatized switchgear wiring: an outline of the WIRES experiment results. In: Grau, A., Morel, Y., Puig-Pey, A., Cecchi, F. (eds.) Advances in Robotics Research: From Lab to Market (2020)

17. Palli, G., Pirozzi, S.: A tactile-based wire manipulation system for manufacturing applications. Robotics (2019)

18. Pirozzi, S., Natale, C.: Tactile-based manipulation of wires for switchgear assembly. IEEE/ASME Trans. Mechatron. (2018)

19. Rittal AG Homepage. https://www.rittal.com/ch-de/content/de/unternehmen/presse/pressemeldungen/pressemeldung_detail_30615.jsp (2014). Accessed 6 Sept 2020

20. Shirakawa, T., Matsuno, T., Yanou, A., Minami, M.: String shape recognition using enhanced matching method from 3D point cloud data. In: IEEE/SICE International Symposium on System Integration (SII) (2015)

21. System Robot Automazione Srl Homepage. https://www.systemrobot.it/en/crlines/wiring-syndy . Accessed 6 Sept 2020

22. Tempel, P., Eger, F., Verl, A.: Reichlich Potential zur Effizienzsteigerung. https://www. schaltschrankbau-magazin.de/artikel/reichlich-potential-zur-effizienzsteigerung/2/. Accessed 7 Sept 2020

23. Ulrich, M.: 3D-Image-Stitching für roboterbasierte Messsysteme. Dissertation, Technical University of Munich (2018)

24. Wnuk, M., Pott, A., Xu, W., Lechler, A., Verl, A.: Concept for a simulation-based approach towards automated handling of deformable objects-A bin picking scenario. In: International Conference on Mechatronics and Machine Vision in Practice (2017)

25. Yumbla, F., Yi, J.-S., Abayebas, M., Shafiyev, M., Moon, H.: Tolerance dataset: mating process of plug-in cable connectors for wire harness assembly tasks. Intel. Serv. Robotics (2020) 
Open Access This chapter is licensed under the terms of the Creative Commons Attribution 4.0 International License (http://creativecommons.org/licenses/by/4.0/), which permits use, sharing, adaptation, distribution and reproduction in any medium or format, as long as you give appropriate credit to the original author(s) and the source, provide a link to the Creative Commons license and indicate if changes were made.

The images or other third party material in this chapter are included in the chapter's Creative Commons license, unless indicated otherwise in a credit line to the material. If material is not included in the chapter's Creative Commons license and your intended use is not permitted by statutory regulation or exceeds the permitted use, you will need to obtain permission directly from the copyright holder. 